Some further particulars of the discovery are given by the New York correspondent of the Times in the issue for Mar. 15. Quoting an announcement which had been received there from the Lowell Observatory, it is stated that the planet was discovered on Jan. 21 on a plate taken with the Lawrence Lowell telescope ; it has since been carefully followed, having been observed photographically by Mr. C. O. Lampland with the large Lowell reflector, and visually with the 24 -inch refractor by various members of the staff. The observers estimate the distance of the planet from the sun as 45 units, which would give a period of 302 years, and mean annual motion of 1.2 degrees.

At discovery, the planet was about a week past opposition, and retrograding at the rate of about $1^{\prime}$ per day ; this has now declined to $\frac{1}{2}^{\prime}$ per day, and the planet will be stationary in April. It should be possible to follow it until the middle of May, when the sun will interfere with observation until the autumn.

The details of the Lowell Observatory positions have not yet come to hand; when they do, it will be possible to derive sufficiently good elements to deduce ephemerides for preceding years. There are many plates that may contain images of the planet ; those taken by the late Mr. Franklin Adams in his chart of the heavens, those taken of the region round Jupiter some twelve years ago for the positions of the outer satellites, and those taken at Königstuhl and elsewhere in the search for minor planets; these all show objects down to magnitude 15 . If early images should be found, they will accelerate the determination of good elements of the new planet; in the case of Uranus, observations were found going back nearly a century before discovery, and in that of Neptune they went back fifty-one years. In the present case, forty years is the most that can be hoped for, and probably very few photographs showing objects of magnitude 15 are available before the beginning of this century.

One of the most difficult problems will be to find the mass of the new body; in Neptune's case, Lassell discovered the satellite a few months after the planet was found, and the mass was thus determined. It is to be feared, however, that the new planet would not have any satellite brighter than magnitude 21. Stars of this magnitude have been photographed with the 100 -inch reflector at Mount Wilson, but it is doubtful whether it could be done within a few seconds of arc of a much brighter body. Failing the detection of a satellite, the mass can only be deduced from a rediscussion of the residuals of Uranus and Neptune; new tables of these planets will ultimately be called for, but that task must wait until the orbit of the new body is known fairly exactly.

The perturbations of Halley's comet will also require revision; at each of the last two returns, there has been a discordance of two or three days between the predicted and observed dates of perihelion passage ; it will be interesting to see whether the introduction of the perturbations of the new body effects an improvement. The late Mr. S. A. Saunder made the suggestion at the time of the last apparition of the comet that an unknown planet might be the cause of the discordance, but it was not then possible to carry the suggestion further. The discovery of a new planet therefore opens a large field of work for mathematical astronomers. It will also appeal to students of cosmogony ; Sir James Jeans, in an article in the Observer for Mar. 16, suggests that it may represent the extreme tip of the cigar-shaped filament thrown off from the sun by the passage of another star close to it. It would have been the first planet to cool down and solidify ; he says, "As a consequence of this, it will probably prove to be unattended by satellites."

\title{
Lowell's Prediction of a Trans-Neptunian Planet.
}

By Dr. J. JACKson.

THE reported discovery of a planet exterior to 1 Neptune naturally arouses the interest of the general public. It will be of importance in theories concerning the genesis of the solar system as to how far it falls into line with the other planets as regards distance, mass, eccentricity and inclination of orbit, and presence or absence of satellites. Its physical appearance will be beyond observation. To those interested in dynamical astronomy, it may be of some interest to consider the data which led to its discovery and to make some comparison with the corresponding facts relating to Neptune.

If the planet which has been reported approximately follows the orbit predicted by Dr. Percival Lowell, the prediction and the discovery will demand the highest admiration which we can bestow. It is true that the problem as regards its general form is a repetition of that solved by Leverrier, Adams, and Galle more than eighty years ago; but its practical difficulty is of quite a different order of magnitude. In short, this discovery, if it turns out to be actually Lowell's pre- dicted planet, was extremely difficult-while Neptune was in fact crying out to be found. Let us look at the actual data.

Uranus was discovered in 1781 by Herschel. Scrutiny of old records showed that it had been observed about a score of times dating back to 1690. The fact that Lemonnier observed it eight times within a month, including four consecutive days, without detecting its character, should be a lesson to anyone who makes observations without examining them. In 1820 Bouvard found that the old and the new observations could not be reconciled, and in constructing his tables boldly rejected the early observations, but the tables rapidly went from bad to worse; the residuals amounted to $20^{\prime \prime}$ in $1830,90^{\prime \prime}$ in 1840 , and to $120^{\prime \prime}$ in 1844 . Adams used in his first approximation data up to 1840, Leverrier data up to 1845. Now Uranus had passed Neptune in 1822. As the relative motion is about $2^{\circ}$ a year, it means that for most of the time covered by the prediscovery observations the perturbations were very small, while from the fact 
that the difference between the heliocentric distances is much smaller than expected from Bode's law, the perturbations at the time of conjunction were relatively large. Consequently the prediction of the longitude of the disturbing body was very easy, while the determination of the other elements were correspondingly difficult. The fact was that the simple hypothesis of the existence of an exterior planet with any sort of guess as to size and shape of orbit would suffice to predict the longitude. In other words, most of the residuals could be closely satisfied provided that substantially correct values of the longitude of the planet and its attractive force $m\left(\frac{1}{\Delta^{2}}-\frac{1}{r^{2}}\right)$ were used. Both Leverrier and Adams easily found values of these quantities, and Galle had no difficulty in detecting the planet.

We now turn to Lowell's " Memoir on a TransNeptunian Planet", published in 1915. The observational basis is the outstanding residuals in the motion of Uranus during two centuries, that is, rather more than two revolutions of that planet round the sun, of somewhat less than two revolutions relative to the predicted planet and of about one relative to Neptune. The following are the values of the observed residuals of Leverrier's and of Gaillot's theories taken from Lowell's memoir.

\begin{tabular}{|c|c|c|c|c|c|}
\hline & Leverrier. & Gaillot. & & Leverrier. & Gaillot. \\
\hline 1709 & & $+2 \cdot 14^{\prime \prime}$ & 1855 & & $-0.50^{\prime \prime}$ \\
\hline 1753 & $+5 \cdot 52^{\prime \prime}$ & $+4 \cdot 45$ & 1858 & $+0.50^{\prime \prime}$ & $-0 \cdot 20$ \\
\hline 1769 & $+4 \cdot 77$ & $+2 \cdot 47$ & 1861 & & -0.36 \\
\hline 1783 & $-3 \cdot 30$ & -0.96 & 1864 & +0.25 & +0.18 \\
\hline 1787 & $-5 \cdot 12$ & $-1 \cdot 20$ & 1867 & & $+1 \cdot 20$ \\
\hline 1792 & $-3 \cdot 50$ & $+0 \cdot 10$ & 1870 & -0.50 & $+1 \cdot 32$ \\
\hline 1796 & -1.88 & -0.69 & 1873 & & +0.75 \\
\hline 1803 & $+0 \cdot 40$ & $-1 \cdot 19$ & 1876 & $-1 \cdot 65$ & -0.50 \\
\hline 1812 & +2.00 & -0.77 & 1879 & & +0.58 \\
\hline 1817 & +0.50 & $-0 \cdot 60$ & 1882 & $-2 \cdot 88$ & +0.52 \\
\hline 1820 & -0.75 & $-2 \cdot 37$ & 1885 & - & -0.17 \\
\hline 1827 & $-2 \cdot 10$ & $+2 \cdot 00$ & 1888 & $-4 \cdot 22$ & -0.85 \\
\hline 1837 & $-1 \cdot 10$ & $-1 \cdot 22$ & 1891 & & $-1 \cdot 11$ \\
\hline 1840 & +0.63 & +0.78 & 1894 & $-5 \cdot 63$ & -0.50 \\
\hline 1843 & & +0.74 & 1897 & & +0.35 \\
\hline 1846 & +0.38 & $-1 \cdot 40$ & 1900 & $-4 \cdot 32$ & +1.00 \\
\hline 1849 & & $-0 \cdot 25$ & 1903 & $-3 \cdot 00$ & +0.65 \\
\hline \multirow[t]{2}{*}{1852} & $-1 \cdot 17$ & -0.95 & 1907 & $\ldots$ & +0.25 \\
\hline & & & 1910 & & $+1 \cdot 10$ \\
\hline
\end{tabular}

The residuals show remarkable differences between the two theories, but Lowell deduced that the residuals exceeded their probable errors four or five times. The problem was to find from these residuals corrections to the elements of the orbit and to find the mass and the elements of the disturbing body. It might almost appear hopeless when we consider that the residuals must be affected by errors in the accepted masses of the known planets. There can be no doubt, however, that the masses adopted by Gaillot for Jupiter, Saturn, and Neptune are very accurate. Lowell's procedure was to adopt a value of the semimajor axis of the unknown body, and a complete series of values for its longitude, and then select the value of the longitude for which the sum of the squares of the residuals was a minimum. The process was repeated with various values of the mean distance until values of the variables were found giving minimum residuals. The process was of course very laborious, but Lowell carried it through with great perseverance. The following extract from his final summary may be quoted : "By the most rigorous method, that of least squares throughout, taking the perturbative action through the first powers of the eccentricities, the outstanding squares of the residuals from 1750 to 1903 have been reduced 71 per cent by the admission of an outside disturbing body."

The inclusion of further terms, of additional years and of the squares of the eccentricity, do not alter the results by any substantial amount. Lowell considered that the remaining irregularities could be explained by errors of observation. No trustworthy results could be found from the residuals in latitude so that the inclination of the orbit to the ecliptic could not be deduced, but Lowell considered that it might be of the order of $10^{\circ}$.

As the solution really depends on the difference of the attraction of the unknown planet on Uranus and on the sun, there are two possible solutions in which the longitudes differ by about $180^{\circ}$. The following elements are for the solution satisfying most nearly the position of the newly found body.

Heliocentric longitude on 1914, July . . $\quad 84.0^{\circ}$ Semimajor axis $\quad \cdot \quad \cdot \quad . \quad 5 \quad 43 \cdot 0$ Mass in terms of the sun's mass . . . 1/50,000 Eccentricity . . . . . $\quad . \quad 0.202$ Longitude of perihelion : $\quad . \quad$. $\quad$. $203.8^{\circ}$

This gives the longitude at the present time as about $104^{\circ}$ compared with $107^{\circ}$ of the new planet. The predicted magnitude was 12 to 13 or about ten times brighter than the observed; and a disc of more than $1^{\prime \prime}$ was predicted. This is a rather serious discordance.

The smallness of the residuals indicated that the forces were small. The mass given above is only 0.4 of the mass of Neptune. At mean conjunction, the attraction of the predicted planet on Uranus would be only one-fifteenth of the attraction of Neptune in a similar position, and in addition it would last for a shorter time on account of the more rapid relative motion.

The discovery of a minor planet of the fifteenth magnitude is an everyday occurrence. The planet reveals itself by a decided motion relative to the stars in the course of taking a photograph. For a planet in the predicted orbit, the motion shown (mostly due to the earth's motion) would in the most favourable circumstances not be more than $2^{\prime \prime}$ or $3^{\prime \prime}$ an hour, and it would probably need a trail of at least $5^{\prime \prime}$ for the planet to be detected. On the other hand, photographs taken on successive days would show decided motion, but the labour of finding the planet in a region containing many thousands of stars from separate photographs would be very great. Probably the Lowell observers have come across several minor planets before they were rewarded by the discovery of the very distant planet.

Astronomers all the world over will naturally look forward with great interest to see how nearly the newly discovered body moves in the orbit predicted by Lowell, and are anxiously waiting for further details of the observations. 